\title{
Multiple Myeloma: A retrospective Analysis of the Patients Treated at Ain Shams University Clinical Oncology Department with a Review of the Literature
}

\author{
Khaled Abdel Karim, Mohamed Yassin Mostafa, Khaled Kamal El-Din Elghoniemy, Moushira Esmail \\ Hassan El-Sakka \\ Department of Clinical Oncology and Nuclear Medicine, Faculty of Medicine - Ain Shams University \\ Corresponding author: Moushira E.H. El-Sakka, Mobile: 01015134301; Email: moushiraesmail@ yahoo.com
}

\begin{abstract}
Background: multiple myeloma (MM) is a malignant neoplasm of plasma cells that accumulate in bone marrow leading to bone destruction and marrow failure. Multiple myeloma accounts for about $1.8 \%$ of all cancers and slightly over $17 \%$ of all the hematologic malignancies in the United States. It is more common in men and for unknown reasons.

Objective: this study aims at analysis the epidemiological data of the patients treated from multiple myeloma at Ain Shams University together with reviewing the different lines of management according to recent recommendations.

Patients and Methods: this retrospective analysis of 62 patients with multiple myloma data recorded at their files with follow up and reviews of the recent advances in the management of multiple myeloma.

Results: we found that $96.8 \%$ of patients showing clinical improvement after treatment on other hand only $3.2 \%$ deteriorated, 61.35 of patients were alive, $9.7 \%$ died and $29 \%$ lost follow up, the mean time to DFS was 22.55 months, mean OS was 63.2 months with $87.8 \%$ of patients survived at the end of the study, as regard mean PFS was 54.9 months with PFS at end of study was $74.9 \%$ of patients, there was insignificant differences between OS and demographic data, laboratory studies, there was insignificant differences between PFS and demographic data, laboratory studies.

Conclusion: Multiple myeloma (MM) is a heterogeneous hematologic malignancy involving the proliferation of plasma cells derived by different genetic events contributing to the development, progression, and prognosis of this disease.
\end{abstract}

Key words: Multiple myeloma, Kahler's disease, Egyptian patients, prognostic factors.

\section{INTRODUCTION}

Multiple myeloma (MM) is a malignant neoplasm of plasma cells that accumulate in bone marrow leading to bone destruction and marrow failure ${ }^{(1)}$.

Most cases of multiple myeloma also feature the production of a para protein (an abnormal antibody) which can cause kidney problems. Bone lesions and hypercalcemia are also often encountered ${ }^{(2)}$.

Multiple myeloma accounts for about $1.8 \%$ of all cancers and slightly over $17 \%$ of all the hematologic malignancies in the United States. Myeloma is most frequently diagnosed among people aged from 65 to 74 years with median age 69 years. About 30,280 new myeloma cases have been estimated in the United States in 2017 with an estimated 12,590 deaths ${ }^{(1)}$.

It is more common in men and for unknown reasons, is twice as common in African Americans as it is in White Americans. With conventional treatment, median survival is 3-4 years, which may be extended to 5-7 years or longer with advanced treatments ${ }^{(3)}$.

\section{AIM OF THE WORK}

This study aims at analysing the epidemiological data of the patients treated from multiple myeloma at Ain Shams University together with reviewing the different lines of management according to recent recommendations.

\section{PATIENTS AND METHODS}

After approval of the local ethics committee, no need for informed consent forms from the patients as the study has no risk of harm to any of the study subjects.

We retrospectively reviewed the records of 62 consecutive patients with MM who treated at Ain Shams University clinical oncology department during the period from January 2011 to December 2015 . The data recorded at their files with follow up and reviews of the recent advances in the management of multiple myeloma.

The patients were analyzed with respect to the demographic profile, staging system, diagnosis, treatment details and survival outcome. Cytogenetic analysis was not performed routinely in our institution. 
Diagnosis of symptomatic multiple myeloma was done based on The International Myeloma Working Group criteria for the diagnosis of $\mathrm{MM}^{(4)}$.

Patients were staged according to DurieSalmon staging system (SDS) or international staging system (ISS) ${ }^{(5)}$, or both whenever possible.

Response to treatment was assessed according to the International Myeloma Working Group (IMWG) uniform response criteria ${ }^{(6)}$.

\section{Statistical methods:}

Patient characteristics were summarized using descriptive statistics. Overall survival (OS) was calculated from date of diagnosis to date of death or date of lost follow up. While Progression free survival (PFS) was calculated from the date of first induction treatment to the date of disease progression or death. Comparisons at the univariate level were made using the log-rank test, and multivariate analysis was performed using a Cox proportional hazards model.

\section{RESULTS}

Table (1): Distribution of the studied cases according to demographic and clinical data $(n=62)$.

\begin{tabular}{|l|c|c|}
\hline \multicolumn{1}{|c|}{ Age } & No. & \% \\
\hline$<50$ & 9 & 14.5 \\
\hline $50-59$ & 20 & 32.3 \\
\hline $60+$ & 33 & 53.2 \\
\hline Min. - Max. & \multicolumn{2}{|c|}{$37.0-74.0$} \\
\hline Mean \pm SD. & \multicolumn{2}{|c|}{$59.0 \pm 8.78$} \\
\hline
\end{tabular}

Table (2): Presenting symptoms.

\begin{tabular}{|l|c|c|}
\hline \multicolumn{1}{|c|}{ Presenting symptoms } & No. & \% \\
\hline Bone ache & 47 & 76 \\
\hline Fatigue & 16 & 26 \\
\hline Muscle pain & 2 & 3.2 \\
\hline Lower limb weakness & 6 & 9.7 \\
\hline Pathological fracture & 8 & 13 \\
\hline Dyspnea & 2 & 3.2 \\
\hline Chest wall swelling & 1 & 1.6 \\
\hline Generalized swelling & 6 & 9.7 \\
\hline Shortness of breath & 2 & 3.2 \\
\hline Incidental discovered & 4 & 6.5 \\
\hline
\end{tabular}

Table (3): Distribution of the studied cases according to Stage at time of presentation $(n=62)$.

\begin{tabular}{|l|c|c|}
\hline Stage at time of presentation & No. & \% \\
\hline I & 20 & 32.3 \\
\hline II & 14 & 22.6 \\
\hline III & 28 & 45.2 \\
\hline
\end{tabular}

Table (4): Number of cycles.

\begin{tabular}{|c|c|c|}
\hline Number of cycles & No. & $\%$ \\
\hline 2 & 4 & 6.5 \\
\hline 3 & 8 & 13 \\
\hline 4 & 2 & 3.2 \\
\hline 6 & 46 & 74 \\
\hline 9 & 2 & 3.2 \\
\hline$\geq 6$ & \multicolumn{2}{|c|}{$2.0-9.0$} \\
\hline Min. - Max & \multicolumn{2}{|c|}{$5.39 \pm 1.51$} \\
\hline Mean \pm SD
\end{tabular}

Table (5): Response to first line and second line.

\begin{tabular}{|l|c|c|}
\hline & No. & \% \\
\hline Response to first line & & \\
SD (stationary disease) & 29 & 47 \\
PR (partial response) & 13 & 21 \\
CR (complete response) & 10 & 16 \\
PD (progressive disease) & 10 & 16 \\
\hline Response to second line & & \\
SD (stationary) & 7 & 28 \\
PR (partial response) & 6 & 24 \\
CR (complete response) & 6 & 24 \\
PD (progression disease) & 6 & 24 \\
\hline
\end{tabular}

Table (6): Distribution of the studied cases according to Toxicities $(n=62)$.

\begin{tabular}{|l|c|c|}
\hline & No. & \% \\
\hline Nausea & 50 & 80.6 \\
\hline Grade 2 & 48 & 77.4 \\
\hline Grade 3 & 2 & 3.2 \\
\hline Peripheral neuropathy & 43 & 69.4 \\
\hline Grade 2 & 24 & 38.8 \\
\hline Grade 3 & 19 & 30.6 \\
\hline Constipation & 11 & 17.7 \\
\hline Grade 2 & 7 & 11.3 \\
\hline Grade 3 & 4 & 6.4 \\
\hline Peripheral oedema & 8 & 12.9 \\
\hline Grade 2 & 8 & 12.9 \\
\hline Grade 3 & 0 & 0.0 \\
\hline Renal impairment & 8 & 12.9 \\
\hline
\end{tabular}

Table (7): Distribution of the studied cases according to Clinical Response $(n=62)$.

\begin{tabular}{|c|c|c|}
\hline Clinical Response & No. & \% \\
\hline Improved & 60 & 96.8 \\
\hline Deteriorated & 2 & 3.2 \\
\hline
\end{tabular}

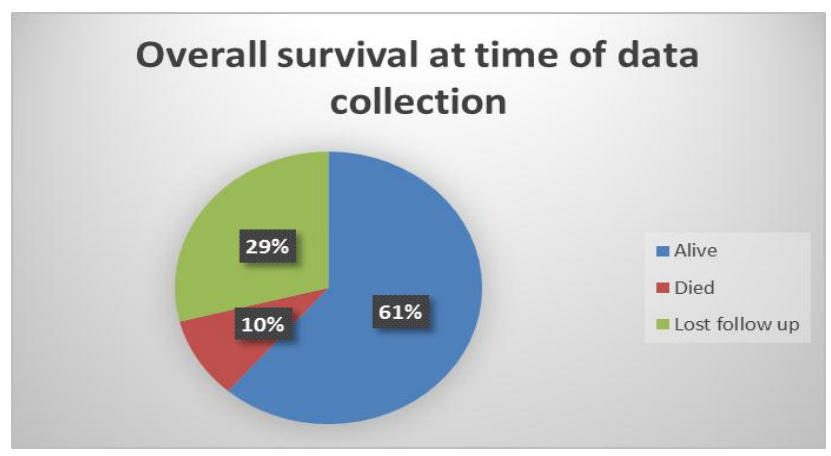

Figure (1): Overall survival at time of data collection. 


\section{DISCUSSION}

Multiple myeloma (MM) is a malignant neoplasm of plasma cells that accounts for about $1.8 \%$ of all cancers and slightly over $17 \%$ of all the hematologic malignancies in the United States. About 30,280 new myeloma cases have been estimated in the United States in 2017 with an estimated 12,590 deaths ${ }^{(1)}$.

In the current study we aimed at having a retrograde view of the Multiple myeloma disease to describe clinic-epidemiological criteria of our patients, describe our results and to improve these results in the future.

The median age of the Egyptian Multiple myeloma patients tend to be generally younger, in our study 62 patients with multiple myeloma was included with a mean age of the involved patients of 59 years, range (37-74 years) with male predominance $(67.7 \%)$, a similar range reported in a retrospective analysis done by Mattar et al. ${ }^{(7)}$ with mean age of 58.5 years (range, 27-80 years). In Saudi Arabian and Moroccan studies, the median ages were 56 years, 59 years, respectively ${ }^{(7)}$.

In the present study we found that the most common presenting symptoms were bone ache (representing 75.8\%), fatigue (representing 25.8\%) and pathological fracture (representing 12.9\%). 69.4\% of patients had anemia, renal impairment in $16.1 \%$ of patients, hypercalcemia in $12.9 \%$ of patients, hypoalbominemia in $38.7 \%$ of patients. As regard beta2- microglobulin its level was increased in $80.6 \%$ of patients and $64.5 \%$ of patients had positive Bence jones protein in urine, $64.5 \%$ of patients had positive SIFE, all patients had biopsy proven plasmacytoma with $96.8 \%$ of patients had confirmatory IHC.

While in a study by Shin $\boldsymbol{e t}$ al., they found that among studied patients $29 \%$ of patients had anemia, 23\%, had hypercalcemia (above $10 \mathrm{mg} / \mathrm{dL}$ ) and $28 \%$ had hypoalbumenimia (less than 3.5 $\mathrm{g} / \mathrm{dL}), 13 \%$ had renal impairement, $48 \%$ had elevated beta2-microglobulin and 65\% had biopsyproven plasmacytoma ${ }^{(8)}$.

In Terebelo et $\boldsymbol{a l} .{ }^{(9)}$, study they found that studied patients had hypercalcemia $(14.7 \%)$, low serum albumin levels (65.2\%), and elevated beta2microglobulin concentrations $(5.5 \mathrm{mg} / \mathrm{L} ; 66.7 \%)^{(9)}$.

In this study we found that the staging of patients according to International Staging System
(ISS) and Durie-Salmon Staging System (DSS) ${ }^{(5)}$, $32.3 \%$ had stage I, $22.6 \%$ had stage II and $45.2 \%$ of patients had disease stage III. While another study by Krejci et al. ${ }^{(\mathbf{1 0})}$, showed that clinical stages at the start of chemotherapy according to DS2 were as follows: stage I in 16 patients (12\%), stage II in 17 cases (13\%) and stage III in 100 cases (75\%). Among the 100 patients with DS III, there were 94 patients with multiple osteolytic lesions $^{(10)}$.

In the present study the cases were diagnosed initially by hypercalcemia, renal failure, anemia and bone ache then we proceede to laboratory investigations as SPEP, SIFE, UPEP, UIFE, Bence John's protein in urine, beta2- microglobulin, bone marrow aspirate and biopsy and IHC also we depend on radiographs in diagnosis like skeletal survey, CT Scan, MRI and PET/CT Scan.

This explained by Rajkumar $\boldsymbol{e t}$ al. (11) $^{\mathbf{1 1}}$ as they demonstrated that the diagnosis of multiple myeloma requires the presence of one or more myeloma defining events (MDE) in addition to evidence of either $10 \%$ or more clonal plasma cells on bone marrow examination or a biopsy-proven plasmacytoma. Myeloma defining events consists of established CRAB (hypercalcemia, renal failure, anemia, or lytic bone lesions) features as well as 3 specific biomarkers: clonal bone marrow plasma cells $\geq 60 \%$, serum free light chain (FLC) ratio $\geq 100$ (provided involved FLC level is $\geq 100 \mathrm{mg} / \mathrm{L}$ ), and more than one focal lesion on magnetic resonance imaging (MRI).Each of the new biomarkers is associated with an approximately $80 \%$ risk of progression to symptomatic end-organ damage in two or more independent studies ${ }^{(11)}$.

The updated criteria represent a paradigm shift since they allow early diagnosis and initiation of therapy before end-organ damage. When multiple myeloma is suspected clinically, patients should be tested for the presence of $M$ proteins using a combination of tests that should include a serum protein electrophoresis (SPEP), serum immunofixation (SIFE), and the serum free light chain (FLC) assay. Approximately $2 \%$ of patients with multiple myeloma have true non-secretory disease and have no evidence of an M protein ${ }^{(11)}$.

According to this study about radiographs we found that $74.2 \%$ had positive skeletal survey, $71 \%, 75.8 \%, 69.4 \%$ had CT, MRI and bone scan respectively but only $9.7 \%$ had PET-CT scan. 
Although in a study by Rajkumar SV they found that plain radiographs of the skeleton are typically required to assess the extent of bone disease, low dose whole body CT, PET/CT and MRI scans are more sensitive and one or more of them are indicated when symptomatic areas show no abnormality on routine radiographs, when there is doubt about the true extent of bone disease on plain radiographs alone, and when solitary plasmacytoma or SMM are suspected ${ }^{(11)}$.

In the present study we found that the regimens that had been used in treatment were one of the following regimens: Melphlan/ prednisone, Thalidomide/ Dexamethasone, Thalidomide/ Endoxan/ Dexamethasone, Vincrestine/ Doxorubcin/ Dexamethasone, Bortezomib/ Endoxan/ Dexamethasone, Bortezomib/ Thalidomide/ Dexamethasone, Bortezomib/ Cyclophospamid/ Dexamethasone. And the mean number of cycles first line treatment was 5.39 cycles with $46.8 \%$ of patients had SD, 21\% had PR, 16.1\% had CR and $16.1 \%$ had PD while the mean number of cycles of second line of treatment was 5 cycles with $28 \%$ of patients had SD,24\% had PR,CR,PD for each. The best outcome regimens as frst line were Bortezomib / Endoxan /Dexa, Bortezomib / thalidomide /Dexa (VTD) while VCD had the best outcome as second line of treatment.

Shin et $\boldsymbol{a l} .{ }^{(8)}$, found that either vincristine/ doxorubicin/ dexamethasone (VAD) alone or two cycles of VAD subsequently followed by ortezomib/ thalidomide/ dexamethasone (VTD) was the most frequently used regimen, followed by thalidomide/ dexamethasone (TD). Among the patients whose response to the first-line treatment could be evaluated, either a complete response (CR) or a very good partial response (VGPR) was achieved in 14 patients (48\%) after first-line chemotherapy with or without subsequent ASCT. Of these patients, five were not eligible for ASCT because of early progression before stem cell collection $(n=2)$, the patient's refusal $(n=2)$, and suicide immediately after the initial response evaluation $(n=1)^{(8)}$.

Regarding the 26 patients with relevant data, 18 (69\%) eventually experienced progressive disease (PD). Bortezomib/ dexamethasone (VD) with or without other agents (such as thalidomide, lenalidomide, doxorubicin, or cyclophosphamide) was administered in 16 patients, of whom $31 \%$ achieved CR or VGPR, 25\% achieved partial response (PR), 25\% achieved stable disease, and $19 \%$ achieved PD. Only three of these patients received a bortezomib-containing regimen as a first-line treatment ${ }^{(8)}$.

In this study almost all patients received palliative radiotherapy while according to Shin study seven patients out of 26 patients received radiotherapy for the palliation of symptoms ${ }^{(8)}$.

Regarding toxicities of chemotherapy in this study, the toxicities of treatment and grading according to Common Terminology Criteria for Adverse Effects (CTCAE) Version 4.0 and it was found that $80.6 \%$ of patients had nausea most of them grade $2,69.4 \%$ had peripheral neuropathy $17.7 \%$ had constipations, $12.9 \%$ had peripheral oedema and $12.9 \%$ had renal impairment.

According to multiple myeloma research foundation (MMRF) ${ }^{(12)}$, the most common side effects of myeloma treatment are nausea, peripheral neuropathy, deep vein thrombosis (DVT), bone marrow depression, conistipation and diarrhea ${ }^{(12)}$.

At the end of the present study we found that $61.3 \%$ of patients were alive, $9.7 \%$ died and $29 \%$ lost follow up. The mean time to DFS was 22.55 months and mean OS was 63.2 months with $87.8 \%$ of patients survived at the end of the study, as regard mean PFS was 54.9 months with PFS at end of study was $74.9 \%$ of patients.

Jung $\boldsymbol{e t} \boldsymbol{a l} .{ }^{(13)}$, found that the median followup duration for the patients was 24 months from the time of diagnosis. Ninety-five patients $(44 \%$, 95/217) died during the study period, The OS rate at two years for the total number of patients was estimated at $67 \%$ with a median OS of 45 months (95\% CI, 29-61 months) ${ }^{(13)}$.

In this study we found that there was significant correlation between ASCT and both OS, PFS it increases with patients who underwent ASCT.

A reported retrospective study also suggested that MM patients diagnosed before 40 years of age do not show significant differences in OS after autologous hematopoietic stem cell transplantation (aHSCT) compared to those aged 41 to 65 years ${ }^{(14)}$. In contrast, some large studies suggested that old age is associated with short survival. Therefore, the importance of age as an independent prognostic factor in MM is still unclear ${ }^{(15)}$.

Most importantly, despite many favorable prognostic factors mentioned above such as low stages at diagnosis, good performance status, and a low frequency of tissue injuries, the median OS was only 61 months. This was much shorter than those observed in other two cohorts, one including patients 
aged 41 to 65 (longer than 80.7 months) and the other including patients aged 60 to 65 (89 months) ${ }^{(15)}$.

The relative differences in results between this study and the other studies may be due to the smaller sample size, short follow up period, lost of follow up of patients and a lot of financial obstacles affected type and availability of myeloma treatment at the time period of this study.

\section{CONCLUSION}

There was significant correlation between 1 st line treatment and both OS, PFS p-value 0.001, 0.002 respectively as best outcome regimens were Bortezomib / Endoxan /Dexa and Bortezomib / Thalidomide / Dexa with $100 \%$ survival at end of study. Overall survival had insignificant correlation with 2 nd line treatment $p$-value 0.613 but there was insignificant correlation with PFS as Bortizomib/ Cyclophosphamide/Dexa had best outcome 100\% survival at end of study with p-value 0.025 .

\section{REFERENCES}

1. Siegel RL, Miller KD, Jemal A (2017): Cancer statistics, 2017. CA: a cancer J Clin., 67:7-30.

2. Dimopoulos M, Kyle R, Fermand JP et al. (2011): Consensus recommendations for standard investigative workup: report of the International Myeloma Workshop Consensus Panel 3. Blood, 117(18):4701-4705.

3. Ocio EM, Mateos MV, Maiso $P$ et al. (2008): New drugs in multiple myeloma: mechanisms of action and phase $\mathrm{I} / \mathrm{II}$ clinical findings. Lancet Oncol., 9(12):1157-1165.

4. Rajkumar SV, Dimopoulos MA, Palumbo A et al. (2014): International myeloma working group updated criteria for the diagnosis of multiple myeloma. Lancet Oncol., 15:538-48.

5. Greipp PR, Miguel JS, Durie BG et al. (2005): International staging system for multiple myeloma. Journal of clinical oncology, 23(15):3412-3420.

6. Kumar S, Paiva B, Anderson KC et al. (2016): International Myeloma Working Group consensus criteria for response and minimal residual disease assessment in multiple myeloma. The Lancet Oncology, 17(8): 328-346.

7. Mattar M, El Husseiny NM, Kasem $\mathbf{N}$ et al. (2014): Multiple myeloma: a descriptive study of 217 Egyptian patients. Annals of Hematology, 93(1): 141-145.
8. Shin J, Koh Y, Youk J et al. (2017): Clinicopathological characteristics of extremely young Korean multiple myeloma patients: therapeutic implications. The Korean journal of internal medicine, 32(4): 722-730.

9. Terebelo H, Srinivasan S, Narang M et al. (2017): Recognition of early mortality in multiple myeloma by a prediction matrix.Am $\mathbf{J}$ Hematol., 92:915-923

10. Krejci M (2005): Prognostic factors for survival after autologous transplantation: a single centre experience in 133 multiple myeloma patients. Bone marrow transplantation, 35(2):159.

11. Rajkumar SV (2016): Multiple myeloma update on diagnosis, risk stratification, and management. American journal of hematology, 91(7):719-34.

12. Multiple myeloma research foundation (2010): Common side effects of multiple myeloma treatments. Blood ,115(6): 1113-1120.

13. Jung HA, Jung SH, Richardson PG et al. (2017): Updated analysis of CALGB (Alliance) 100104 assessing lenalidomide versus placebo maintenance after single autologous stem-cell transplantation for multiple myeloma: a randomised, double-blind, phase 3 trial. The Lancet Haematology, 4(9):431-442.

14. Cheema PK, Zadeh S, Kukreti V et al. (2009): Age 40 years and under does not confer superior prognosis in patients with multiple myeloma undergoing upfront autologous stem cell transmplant. Biol Blood Marrow Transplant., 15:686-693.

15. Chretien ML, Hebraud B, Cances-Lauwers V et al. (2014): Age is, a prognostic factor even among patients with multiple myeloma younger than 66 years treated with high-dose melphalan: the IFM experience on 2316 patients. Haematologica, 99:1236-1238. 